\title{
Design Thinking como Metodologia para Definição da Interface Gráfica de Usuário
}

\author{
Daila B. Nunes ${ }^{1}$, Lucieli B. Guerra ${ }^{1}$, Claiton M. Correa ${ }^{1}$ \\ ${ }^{1}$ LarDev - Instituto Federal Farroupilha - (IFFar) \\ São Borja - RS - Brasil \\ daila.beckersb@gmail.com , \{luciele.beque, claiton.correa\}@iffar.edu.br
}

\begin{abstract}
Design Thinking has been gaining attention in recent years as a methodology for creating innovative and creative solutions. We can use it to develop solutions for different areas of knowledge. This article addresses the use of Design Thinking as a methodology to support the development of the user interface of the Cine Web 2.0 project, concerning the inside-out approach of development from HCI research field. The article also presents and briefly discusses the results obtained in the project.
\end{abstract}

Resumo. A técnica de Design Thinking vem ganhando atenção nos últimos anos como metodologia para criar soluções inovadoras e criativas. Ela pode ser empregada para o desenvolvimento de soluções para diversas áreas do conhecimento. Este artigo trata da utilização de Design Thinking como metodologia de apoio ao desenvolvimento da interface de usuário do projeto Cine Web 2.0, considerando a abordagem de desenvolvimento de dentro para fora da ára de IHC. O artigo também apresenta e discute brevemente os resultados obtidos no projeto.

\section{Introdução}

Os sistemas computacionais interativos são construídos a partir de um conjunto de requisitos e executam instruções predefinidas. Do ponto de vista de desenvolvimento, a fim de elucidar os requisitos e construir um software que os atenda, as equipes de desenvolvimento fazem uso de técnicas como entrevistas, brainstorming, entre outras.

O processo de construção de um software envolve um conjunto de stakeholders, sendo que cada um tem uma visão diferente sobre o produto em desenvolvimento. A Interação Humano-Computador (IHC) é uma área multidisciplinar interessada na qualidade de uso desses sistemas e seu impacto na vida dos seus usuários [Junqueira e da Silva 2010].

Simone e Bruno [Junqueira e da Silva 2010] argumentam que para conceber um sistema interativo mais adequado ao mundo em que se está inserido, a IHC adota uma abordagem de desenvolvimento de dentro para fora. Nesta abordagem, conforme os autores, o projeto de um sistema começa investigando os atores envolvidos, seus interesses, objetivos, responsabilidades, motivações, entre outros.

Uma das formas de garantir que o desenvolvimento leve em consideração as atividades dos usuários é envolvê-los ao longo do desenvolvimento [Rogers et al. 2013]. Assim, os desenvolvedores podem obter uma melhor compreensão dos objetivos dos 
usuários. Neste contexto, a metodologia de Design Thinking (DT) surge como uma forma de manter o foco nos usuários durante o desenvolvimento do produto [Ostrowski et al. 2015].

Diante deste cenário, o objetivo deste artigo é apresentar os resultados da adoção da metodologia de DT, com o intuito de propor uma nova interface de usuário para o sistema denominado CineWeb 2.0, desenvolvido para o projeto de extensão Cine Campus. Esse projeto é realizado no Instituto Federal Farroupilha - Campus São Borja há 10 anos.

O artigo está organizado como segue. A Seção 2 apresenta a abordagem adotada para condução do trabalho. A Seção 3 apresenta e discute os resultados obtidos. A Seção 4 traz as considerações finais do trabalho. Por fim, as referências são apresentadas.

\section{Materiais e Métodos}

Design Thinking (DT) é considerado um conjunto de técnicas e ferramentas centrado no usuário que suporta um processo iterativo para produzir e analisar soluções para problemas reais [Junior et al. 2013]. DT não é apenas um motor para inovação promovido pelos designers, mas oferece modelos de processos para serem conduzidos não apenas por designers, mas por equipes multidisciplinares e em quaisquer tipos de organizações [Tschimmel 2012].

A Figura 1 ilustra o modelo de processo de design chamado de duplo diamante adotado neste trabalho. O modelo é constituído por quatro etapas, denominadas: Descobrir, Definir, Desenvolver e Entregar. Em cada uma destas etapas é possível empregar uma ou mais técnicas para amadurecer o conhecimento da equipe de desenvolvimento acerca do produto em construção.

A utilização de DT no processo de design do produto contribui para melhorar a sua imagem e a sensação de bem-estar de futuros usuários, uma vez que estes são considerados especialistas em sua interação com determinados produtos ou serviços [Tschimmel 2012].

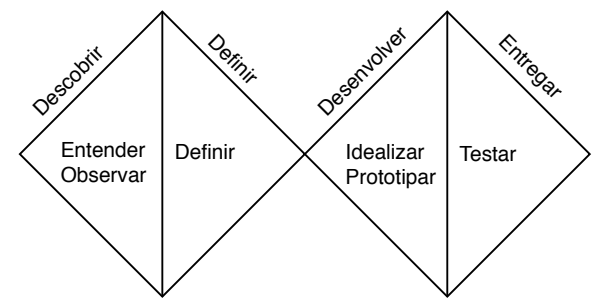

Figura 1. Modelo Duplo Diamante e HPI D-School

De fato, o emprego de DT como forma de promover a inovação e pensamento criativo a fim de definir uma interface de usuário é reportado com sucesso na literatura. Como exemplos, Arne Berger [Berger 2011] argumenta, em seu trabalho, que a técnica pode ser incorporada em qualquer estágio do ciclo de produção, principalmente na etapa de desenvolvimento, através da criação de protótipos. Já a autora Carolina Cavalcanti [Cavalcanti 2014] apresenta o uso de DT como forma de identificar o problema, idealizar possíveis soluções e prototipar um Ambiente Virtual de Aprendizagem (AVA) centrado no usuário. 


\section{Resultados}

O trabalho desenvolvido seguiu o modelo de DT apresentado na Figura 1. Em cada uma das etapas, técnicas como entrevistas e testes de usabilidade foram empregadas a fim de auxiliar o entendimento do problema por parte da equipe de desenvolvimento. $\mathrm{O}$ desenvolvimento de cada etapa é relatado na sequência.

- Descobrir: esta etapa compreendeu a interação da equipe de desenvolvimento com os usuários administradores do sistema, isto é, administrador principal e bolsistas com privilégios de acesso ao sistema. Para entender melhor o problema a técnica de entrevista foi empregada. Com as entrevistas foi possível identificar qual a percepção dos usuários quanto à interface do sistema, seus pontos fortes e fracos. Através das entrevistas as características que melhor representavam o projeto também foram identificadas.

Além da entrevista, nesta etapa foram conduzidos testes de usabilidade do sistema. Os testes compreenderam a execução, pelos usuários, de um conjunto de tarefas rotineiras do sistema e a observação pela equipe que conduziu os testes[Rogers et al. 2013]. Os testes de usabilidade evidenciaram problemas em relação à página inicial do projeto, à disposição de informações referentes à filmes, debatedores, entre outros, bem como menus e comandos relacionados de forma incorreta.

- Definir: nesta etapa, todos os problemas da etapa anterior - Descobrir-foram documentados e classificados com base na Avaliação Heurística [Nielsen 1995]. Nesta etapa foi possível classificar os problemas de usabilidade percebidos com referência as 10 heurísticas propostas.

- Desenvolver: esta etapa compreendeu o desenvolvimento de protótipos, que representam a proposta da equipe para resolver ou reduzir os problemas de usabilidade encontrados na primeira etapa do DT.

- Entregar: esta etapa compreendeu a validação das propostas de interface criadas.

Foi possível observar, por meio das entrevistas diretas com os usuários, problemas de usabilidade que o sistema CineWeb 2.0 possuía e que passavam despercebidos pelos desenvolvedores. No geral, o feedback dos usuários foi positivo, ambos descreveram que os protótipos suprem suas reais necessidades e solucionam os problemas de usabilidade na interface antiga do sistema CineWeb. Os usuários relataram também que o novo protótipo de interface foi muito além do esperado, o que tornou a interface do CineWeb $2.0 \mathrm{um}$ ambiente agradável e fácil de ser utilizado na interação humano-computador.

\section{Considerações finais}

O Design Thinking é bastante aplicado em diversas áreas do conhecimento, e gera soluções que atendem as reais necessidades das pessoas que utilizam um determinado produto. $\mathrm{O}$ mesmo aplicado à construção de interfaces, auxilia na projeção de um sistema diferente dos outros, pois se baseia nos desejos e necessidades dos usuários, tornando o produto final centrado no usuário.

$\mathrm{O}$ resultado percebido pelos autores e o feedback fornecido pelos participantes, após concluída as etapas do processo de design duplo diamante, servem como exemplo da utilidade e impacto que a técnica de DT agrega na criação de soluções. O uso do método possibilitou a identificação de problemas de usabilidade que o sistema CineWeb 
2.0 possuía através do contato direto com os usuários por meio de entrevistas. Estes problemas foram documentados, avaliados e classificados, o que facilitou no processo de criação das soluções para o mesmo. Por conseguinte, foram criadas soluções para todos os problemas encontrados, e desenvolvidos protótipos que materializam estas soluções.

A dinâmica do DT influencia os designers a pensar em situações sob ângulos diferentes, nas quais a empatia ajuda a identificar desafios que em condições normais não seriam percebidos.

A criação de soluções de IHC necessitam de técnicas que gerem afinidade com os usuários e que favoreçam a identificação das suas reais necessidades. Desta forma, o DT auxilia na criação de soluções que causem um impacto positivo para os usuários do qual o projeto está endereçado.

\section{Referências}

Berger, A. (2011). Design thinking for search user interface design. Proceedings of euroHCIR2011, pages 1-4.

Cavalcanti, C. M. C. (2014). Design thinking como metodologia de pesquisa para concepção de um ambiente virtual de aprendizagem centrado no usuário. SIED: EnPED-Simpósio Internacional de Educação a Distância e Encontro de Pesquisadores em Educação a Distância.

Junior, M., Freitas, R., Peres, S., Fantinato, M., Steinbeck, R., e Araújo, U. (2013). Experimenting with design thinking in requirements refinement for a learning management system.

Junqueira, S. D. e da Silva, B. S. (2010). Interação Humano-Computador, volume 1 of 1. Elsevier, Rio de Janeiro, 1 edition.

Nielsen, J. (1995). How to conduct a heuristic evaluation [em linha]. Nielsen Norman Group Web site. Acedido maio, 9:2012.

Ostrowski, S., Rolczyundefinedski, R., Pniewska, J., e Garnik, I. (2015). User-friendly e-learning platform: A case study of a design thinking approach use. In Proceedings of the Mulitimedia, Interaction, Design and Innnovation, MIDI '15, New York, NY, USA. Association for Computing Machinery.

Rogers, Y., Sharp, H., e Preece, J. (2013). Design de Interação: Além da Interação Humano-Computador, volume 1 of 1 . Bookman, Porto Alegre, 3 edition.

Tschimmel, K. (2012). Design thinking as an effective toolkit for innovation. In ISPIM Conference Proceedings, page 1. The International Society for Professional Innovation Management (ISPIM). 